Historic, Archive Document

Do not assume content reflects current scientific knowledge, policies, or practices. 

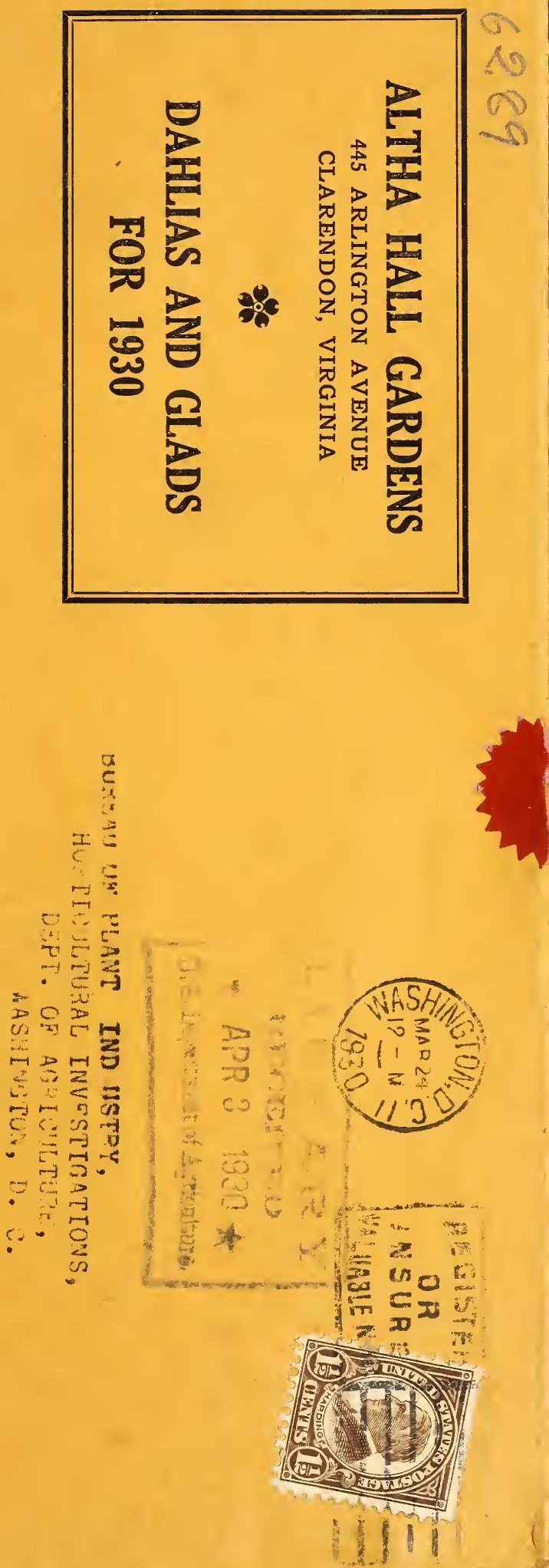


\section{WHOLESALE AND RETAIL}

In order to dispose of our stock of these two flowers, we are offering the following varieties at prices that will prove attractive.

All retail orders prepaid. Wholesale orders F.O.B.

No order for less than $\$ 2.00$ can be accepted.

Reference: Riggs National Bank.

Member: American Dahlia Society, American Gladiolus Society, American Iris Society, American Peony Society.

\section{DAHLIAS}

Tubers sent out are GUARANTEED true to name, free from disease, to have a live eye and be in good growing condition when sent. I cannot be held responsible, if tubers are allowed to dry out before planting. ANY TUBERS PROVING NOT SATISFACTORY WILL BE REPLACED OR MONEY REFUNDED UPON RETURN OF TUBERS.

\section{5 at Hundred Rates}

\begin{tabular}{|c|c|c|c|}
\hline Name and Type & One & Three & Dozen \\
\hline Aimee-Pom. & $\$ .25$ & $\$ .50$ & \\
\hline Alice Whittier-H. C. & 1.50 & 4.00 & \\
\hline Alex. Waldie-Dec. .. & .50 & 1.25 & $\$ 3.60$ \\
\hline Alex. Pope-H. C.... & 2.00 & 5.00 & \\
\hline Altha Hall Crystal-Dec................ & 1.00 & 2.50 & 9.00 \\
\hline Ambassador-Cac. & 1.00 & 2.50 & \\
\hline Amber Glow-Cac. & .50 & 1.00 & \\
\hline Ami Nonin-Col. & .25 & .50 & \\
\hline Arbutus-Pom. ... & .25 & .50 & \\
\hline Attraction-H. C. ....... & .50 & 1.00 & 3.00 \\
\hline Bashful Giant-Dec. .... & .50 & 1.00 & \\
\hline Ben Wilson-Dec. & .75 & 2.00 & \\
\hline Black Diamond-Show ................... & .50 & 1.00 & \\
\hline Bonnie Brae-Dec. & .50 & & \\
\hline Boutonnaire-Pom. & .50 & 1.25 & \\
\hline Break O'Day-H. C. . & .50 & 1.25 & \\
\hline Buddy Benedict-Dec. ................ & 1.00 & 2.50 & $9: 00$ \\
\hline 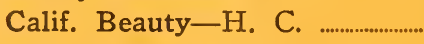 & .50 & 1.25 & 3.60 \\
\hline Camille Frachon-Peo. .................... & .40 & 1.00 & \\
\hline Casper G. Ware-Dec. & 1.00 & 2.50 & \\
\hline Champagne-Dec. & 1.00 & 2.50 & \\
\hline Chas. H. Stratton-Dec. .............. & 1.00 & & \\
\hline Chemar's Eureka-Dec. ............. & 8.00 & & \\
\hline 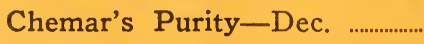 & 3.00 & 8.00 & 30.00 \\
\hline Chemar's 2406-Dec. & 3.00 & & \\
\hline 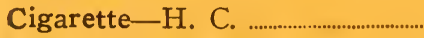 & .75 & 2.00 & 6.00 \\
\hline City of Trenton-Dec. .................. & 4.00 & 11.00 & \\
\hline 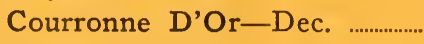 & 1.25 & 3.50 & \\
\hline Delice-Dec. & .35 & 1.00 & \\
\hline Dr. A. L. House-Dec...................... & 1.00 & 2.75 & 9.00 \\
\hline Dr. M. A. Howe-D & 1.00 & & \\
\hline
\end{tabular}


Name and Type

One

Three Dozen

Dresselhuys-Dec.

$.50 \quad 1.00$

Eagle Rock Beauty-Dec.......... $3.00 \quad 8.00$

Earle Williams-Dec. .................. $\quad .75 \quad 2.00$

E1 Dorado-Dec.

$2.00 \quad 6.00$

Eleanor Martin-Dec. ................... 1.50

Ellinor Vanderveer-Dec. ...... $\quad 7.5$

Emma Marie-H. C. ..................... 1.00

$2.00 \quad 6.00$

Emperor-Dec.

$2.50 \quad 6.00$

$\begin{array}{lll}7.5 & 2.00 & 4.80\end{array}$

Esther R. Holmes-H. C. ...... .50

E. T. Bedford-Dec. ..................... $\quad .75$

Flamingo-Dec.

100 for 12.00

Florence Finger-Dec.

.50

Frances Lobdell-H. C. ............ $\quad .75$

Giant Ruby-Dec. ……….................. $\quad .50$

Gladys Sherwood-H. C. ......... .50

Gorgeous-Peo. .................................... $\quad .75$

Herald Tribune Elite-H. C. 1.50

Helen Sparks-Dec.

2.00

His Majesty-H. C. ........................ $\quad .75$

Insulinde-H. C. .................................. $\quad .75$

Jane Cowl-Dec. ............................. $\quad 6.00$

Jean Kerr-Dec.

.35

Jersey's Beacon-Dec.

1.00

100 for 12.00

$2.00 \quad 6.00$

1.25

1.25

5.00

15.00

1.00

100 for 18.00

$\begin{array}{lll}.75 & 2.00 & 7.50\end{array}$

Jersey's Beauty-Dec.

2.00

100 for 30.00

Jersey's Mammoth-Dec. ......... 1.00

2.50

Jersey's Radiant-H. C.

1.00

Jersey's Wonder-Dec.

2.50

John Lewis Child-Dec. ........... $\quad .50$

1.25

Judge Marean-Dec.

.75

J. W. Davies-Dec.

1.00

Kemp's Violet Wonder-Dec. $\mathbf{7 . 5 0}$

King Albert-Dec.

Kohinoor-H. C. .................................. $\quad .75$

La Rhoda-Dec. .................................. $\quad \mathbf{7 . 5 0}$

Lenore-Dec. ......................................... $\quad .35$

Lillian Baldwin-Dec. ................... 1.00

Little Jewell-Pom. ......................... $\quad .75$

Mabel Thatcher-Dec. ............... $\quad .50$

MacGregor-Dec. ............................... $\quad 1.00$

Mae Grefe-Dec. ................................ 1.00

Magruder-Dec. .................................... 2.00

Margaret Masson-Dec. ............. 1.00

Maude Adams-Show .................. $\quad .50$

2.00

Mephistopheles-Dec.

M. H. DeYoung-Dec.

$.50 \quad 1.25$

Mme. C. H. Goode-Cac. ...... 2.50

Mrs. C. D. Anderson-Show

Mrs. Edna Spencer-Cac. ...... . .35

Mrs. F. E. Bullard-Peo............. 1.00 
Name and Type

Mrs. I. D. V. Warner-Dec. $\quad .50$

Mrs. J. T. Scheepers-Dec.... $\quad .75$

Mrs. Macfarland-Dec. ............. $\quad 2.00$

Mrs. R. Lohrman-Dec. ........... $\quad .75$

Mrs. S. T. Mather-Dec............ 2.00

Mrs. W. E. Estes-H. C............ $\quad .50$

Myra Valentine-Dec. ................. $\quad .75$

Nagel's Roem-H. C. ………….... 1.50

Nancy Helen-Dec. ...................... $\quad .50$

Nancy Sue Lang-Dec.............. 3.00

Newport Wonder-Sin. .............. $\quad .75$

Nichu-H. C. .............................. $\quad .50$

Oregon Beauty-Dec. ............... $\quad .50$

Oyster Bay Beauty-Dec.......... 1.00

Pat O'Mara-Dec. ........................... $\quad .50$

Pink Perfection-Dec. ................... $\quad .75$

Pop Stewart-Dec. ……………..... 1.50

Pride of Calif.-Dec. ..................... $\quad .35$

Pride of Conn.-Dec. ................. $\quad .75$

Primula Rex-Dec.

Robert Scott-Dec.

Robert Treat-Dec.

Rollo Boy-H. C.

Roman Eagle-Dec.

Rose Fallon-Dec.

Rose Pink Cent.-Sin.

Ruth Van Fleet-H. C. .............. $\quad .50$

Sagamore-Dec. .................................. $\quad .75$

Sanhican's Gem-Dec. .................. 1.00

Sanhican's Magnate-Dec. ...... 1.50

Senorita-H. C. .............................. $\quad .75$

Shirley Eileen Shattuck ............ 3.00

Signor-H. C. ................................. 2.00

Silverado-H. C. ............................... 2.00

Sole Mio-Dec. …………………..... 1.25

Snowclad-Pom. ................................ $\quad .25$

Snowdrift-Dec. …………………....... $\quad .50$

Sorosis Souvenir-Dec. .............. $\quad .75$

Susan G. Tevis-Dec. ………….. $\quad .75$

Sylvia Dickey-Dec. ………………. $\quad .50$

Tommy Atkins-Dec. ………..... $\quad .75$

U. S. A.-Peo. .................................. $\quad .75$

Venus-Dec.

$.75 \quad 2.00$

$.50 \quad 1.00$

100 for 17.00

Three Dozen

1.25

100 for 15.00

2.00

2.00

$5.00 \quad 18.00$

$1.00 \quad 3.00$

2.00

$1.00 \quad 3.00$

$1.00 \quad 3.00$

2.50

1.00

100 for 15.00

4.00

.75

2.00

4.00

2.00

2.75

2.50

2.50

.50

2.00

2.50

8.00

5.00

2.00

1.00

2.00

12.00

White Century-Sin. .................... $\quad .25$

Waldheim Sunshine-Dec. ...... 6.00

Winfield Slocombe-Dec. ......... $\quad .75$

Wizard of $\mathrm{Oz}$-Dec. ....................... 1.00

World's Best White-Dec....... 1.00

Yellow Colosse-Show .............. $\quad .50$

Ysleta-Dec. ........................................... $\quad .50$ 



\section{GLADIOLI}

The following varieties are offered to close out our stock. They are listed by the dozen. SIX at dozen rate. This is all clean stock and true to name. All large sized bulbs.

40c per dozen

\section{Alice Tiplady}

Halley

Le Marshal Foch

Mrs. Francis King

Topaz

Wilbrink

$60 \mathrm{c}$ per dozen

Albania

Baron J. Hulot

Carmen Sylva

Gold

Ming Toy

Mrs. Dr. Norton

Mrs. F. C. Peters

Mrs. Frank Pendleton

Rose Ash

Scarlet Princeps

Souvenir

\section{$\$ 1.00$ per dozen}

Mrs. Leon Douglas

Muriel

Purple Glory 50c per dozen

Crimson Glow

Dr. Van Fleet

E. J. Shaylor

Elora

Evelyn Kirtland

Lily White

Lucette

Maiden's Blush

Theda

75c per dozen

Giant Nymph

Golden Measure

Los Angeles

Louise

Milady

Opalescent

Parader

Peace

Pink Wonder

Priority

Vanity

Yellow Treasure

\section{$\$ 1.50$ per dozen}

Dr. F. E. Bennet

Rosemary

Scarlet Wonder

Van der Neer

The following varieties are offered by the hundred. 25 at hundred rates.

\begin{tabular}{|c|c|c|c|}
\hline & No. 1 & No. 2 & No. \\
\hline Albania & $\$ 3.00$ & $\$ 2.50$ & $\$ 1.75$ \\
\hline lice Tiplady & 2.00 & 1.75 & 1.25 \\
\hline Crimson Glow & 2.75 & 2.25 & 1.50 \\
\hline Elora …………................... & 3.00 & 2.25 & 2.00 \\
\hline Giant Nymph & 5.50 & 4.75 & 4.00 \\
\hline Golden $\mathrm{Me}$ & 5.00 & 4.00 & 3.00 \\
\hline Los Angeles ............. & 4.00 & 3.00 & 2.00 \\
\hline Lucette & 3.00 & 2.50 & 2.00 \\
\hline Mrs. Pendleto & 3.00 & 2.50 & 2.00 \\
\hline Opalescent & 4.00 & 3.25 & 2.50 \\
\hline Top: & 2.25 & 1.75 & 1.40 \\
\hline Wilb & 2.25 & 1.75 & 1.40 \\
\hline Yellow Trea & 3.00 & 2.50 & 2.00 \\
\hline Mixture & 2.00 & 1.50 & 1.25 \\
\hline
\end{tabular}


3

$\because 6$ 